\title{
Layer-by-Layer Spray-Coating of Cellulose Nanofibrils and Silver Nanoparticles for Hydrophilic Interfaces
}

\author{
Qing Chen,* Calvin J. Brett, Andrei Chumakov, Marc Gensch, Matthias Schwartzkopf, Volker Körstgens, \\ L. Daniel Söderberg, Anton Plech, Peng Zhang, Peter Müller-Buschbaum, and Stephan V. Roth*
}

Cite This: ACS Appl. Nano Mater. 2021, 4, 503-513

Read Online

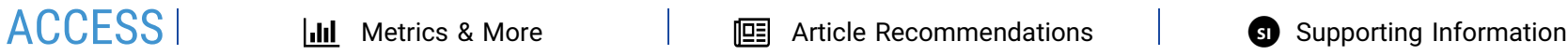

ABSTRACT: Silver nanoparticles (AgNPs) and AgNP-based composite materials have attracted growing interest due to their structure-dependent optical, electrical, catalytic, and stimuliresponsive properties. For practical applications, polymeric materials are often combined with AgNPs to provide flexibility and offer a scaffold for homogenous distribution of the AgNPs. However, the control over the assembly process of AgNPs on polymeric substrates remains a big challenge. Herein, we report the fabrication of $\mathrm{AgNP} /$ cellulose nanofibril (CNF) thin films via layer-by-layer (LBL) spray-coating. The morphology and selfassembly of AgNPs with increasing number of spray cycles are characterized by atomic force microscopy (AFM), grazingincidence small-angle X-ray scattering (GISAXS), and grazing-

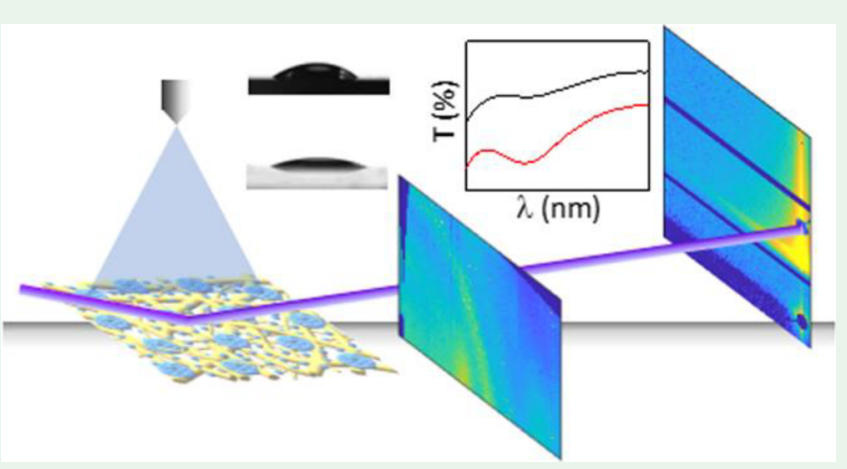
incidence wide-angle X-ray scattering (GIWAXS). We deduce that an individual AgNP (radius $=15 \pm 3 \mathrm{~nm}$ ) is composed of multiple nanocrystallites (diameter $=2.4 \pm 0.9 \mathrm{~nm}$ ). Our results suggest that $\mathrm{AgNPs}$ are assembled into large agglomerates on $\mathrm{SiO}_{2}$ substrates during spray-coating, which is disadvantageous for AgNP functionalization. However, the incorporation of CNF substrates contributes to a more uniform distribution of AgNP agglomerates and individual AgNPs by its network structure and by absorbing the partially dissolved AgNP agglomerates. Furthermore, we demonstrate that the spray-coating of the AgNP/CNF mixture results in similar topography and agglomeration patterns of AgNPs compared to depositing AgNPs onto a precoated CNF thin film. Contactangle measurements and UV/vis spectroscopy suggest that the deposition of AgNPs onto or within CNFs could increase the hydrophilicity of AgNP-containing surfaces and the localized surface plasmon resonance (LSPR) intensity of AgNP compared to AgNPs sprayed on $\mathrm{SiO}_{2}$ substrates, suggesting their potential applications in antifouling coatings or label-free biosensors. Thereby, our approach provides a platform for a facile and scalable production of AgNP/CNF films with a low agglomeration rate by two different methods as follows: (1) multistep layer-by-layer (LBL) spray-coating and (2) direct spray-coating of the AgNP/CNF mixture. We also demonstrate the ability of CNFs as a flexible framework for directing the uniform assembly of AgNPs with tailorable wettability and plasmonic properties.

KEYWORDS: silver nanoparticles, self-assembly, cellulose nanofibrils, layer-by-layer deposition, spray-coating

\section{INTRODUCTION}

Silver nanomaterials have attracted a wide range of interests due to their applications in catalysts (using AgNPs supported on a metal-organic framework for $\mathrm{CO}_{2}$ conversion ${ }^{1}$ and AgNPs deposited on $\mathrm{Fe}_{3} \mathrm{O}_{4}$-coated polydopamine for methylene-blue reduction ${ }^{2}$ ), antimicrobials (including AgNPs deposited on cotton fabrics ${ }^{3}$ and various kinds of textiles ${ }^{4}$ ), sensors (using $\mathrm{Ag}_{2} \mathrm{~S}$ nanoparticles for microRNA sensing ${ }^{5}$ and carbon nanotube/AgNP composite membrane for human-motion detection $^{6}$ ), and electronics (AgNP-based elastic conductors ${ }^{7}$ and electric circuit ${ }^{8}$ ). The tunable performance of AgNP-based devices shows a significant dependence on the structure and morphology of AgNPs at the nanoscale. ${ }^{9-11}$ Polymers, especially cellulose nanofibrils (CNFs), can provide a scaffold for assembly of metallic nanoparticles (MNPs) with defined porosity and roughness, thus increasing the flexibility and ordering of the MNP layer and improving the catalytic, photonic, or electrical performance of the MNP/CNF composite nanomaterials. ${ }^{12-14}$ For example, plasmonic AgNPs were grafted onto cellulose nanocrystals to achieve remote IR actuation, ${ }^{15} \mathrm{AgNP}$ ink was printed on a cellulose nanopaper with high mechanical strength and flexibility, ${ }^{16}$ and dialdehyde nanofibrillated cellulose (DANFC) was used to

Received: October 21, 2020

Accepted: December 17, 2020

Published: January 6, 2021 


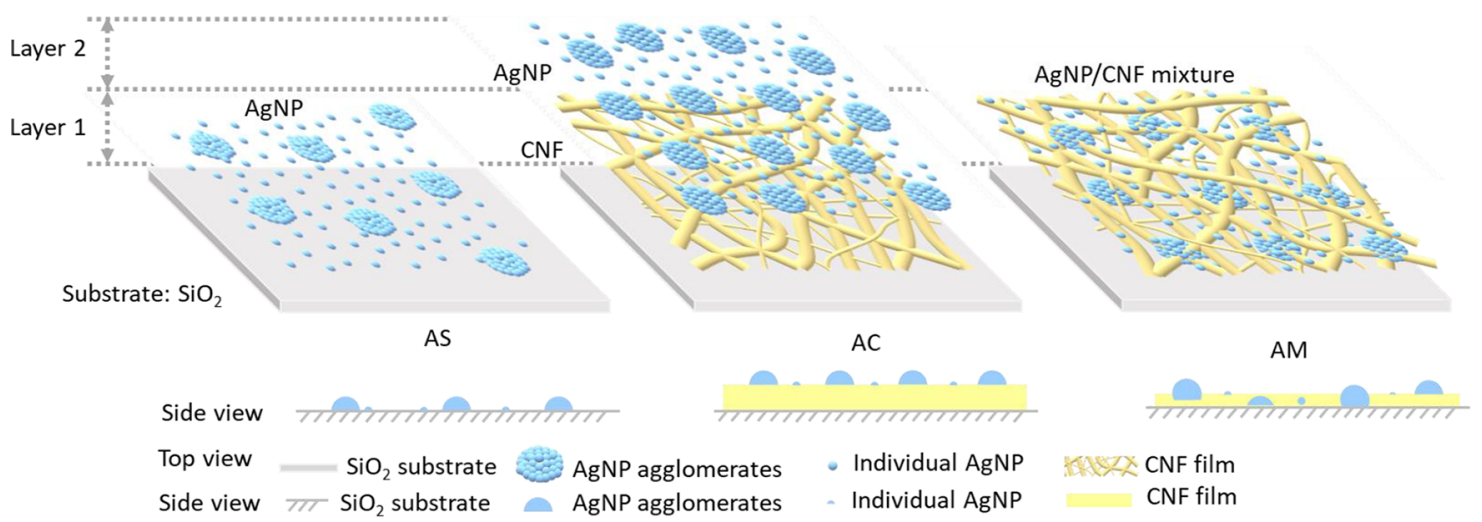

Figure 1. Sketch of the layered structure of the silver nanoparticles (AgNPs) on the $\mathrm{SiO}_{2}$ substrate (the AS samples, left), AgNPs spray-coated onto a cellulose nanofibril (CNF) thin film, which is precoated on a $\mathrm{SiO}_{2}$ substrate (the $\mathrm{AC}$ samples, middle), and a $\mathrm{AgNP} / \mathrm{CNF}$ mixture on the $\mathrm{SiO}{ }_{2}$ substrate (the AM samples, right), respectively.

synthesize a smooth silver layer to obtain a controlled release of antibacterials. ${ }^{17}$ However, the reproducible fabrication of AgNP/cellulose nanocomposites with tailorable morphology remains a challenge, which relies on a nanoscopic characterization of the self-assembly of AgNPs on and/or within cellulose nanomaterials.

Spray-coating is broadly used to fabricate functional multilayered films. ${ }^{18,19}$ Spray-coating allows for (1) controlling the morphology of the deposited layer through adjustable spraying parameters ${ }^{20,21}$ and (2) fabricating composite materials by layer-by-layer (LBL) installation of multilayered films, thus providing a facile approach for assembling multicomponent films with tailorable morphology. ${ }^{22,23}$ Grazing-incidence small-angle X-ray scattering (GISAXS) and grazing-incidence wide-angle X-ray scattering (GIWAXS) are surface sensitive characterization methods that elucidate the structure and morphology of materials at the nanoscale. ${ }^{2,24}$ In the present work, we follow the structural evolution of AgNPs onto or within the CNF layer through the combination of LBL spray-coating and GISAXS/GIWAXS techniques. Three model systems are studied as follows: (1) AgNPs are deposited on $\mathrm{SiO}_{2}$ substrates (AS) to show the assembly of AgNPs on a smooth and nonporous surface, (2) AgNP/CNF LBL film (AC) in which AgNP solution is spray-coated on a CNF thin film, which is deposited on $\mathrm{SiO}_{2}$ substrates in advance, and (3) a $\mathrm{AgNP} / \mathrm{CNF}$ mixture is deposited on $\mathrm{SiO}_{2}$ substrates (AM) to study the self-assembly of AgNP/CNF composite films with AgNPs on and within the CNF network, respectively. AFM is used to visualize the topography of AgNP layers and rationalize the results from GISAXS and GIWAXS. Contact-angle measurements and transmission $\mathrm{UV} /$ vis spectroscopy are employed to demonstrate the potential of $\mathrm{AgNP} / \mathrm{CNF}$ composite films as hydrophilic coatings with plasmonic properties.

\section{METHODS}

Materials and Preparation. The composition and charge density of the chemical groups of CNFs and AgNPs are significant for the intrinsic bond strength between AgNPs and CNFs in their composites. ${ }^{25}$ Therefore, 0.07 wt \% aqueous solution of TEMPOCNF with a surface charge of $800 \mu \mathrm{mol} / \mathrm{g}$ and a radius of around 2.5 $\mathrm{nm}$ was dispersed by sonification and centrifugation ${ }^{26,27}$ to prepare the CNF thin film in the AC samples. An aqueous dispersion of surfactant-free AgNPs with a final concentration of $5 \pm 2 \mu \mathrm{g} / \mathrm{mL}$ (Figure S1) and a zeta potential of $-27.5 \pm 2.5 \mathrm{mV}^{28}$ was produced by pulsed-laser ablation ${ }^{29}$ to prepare the AgNP layer in the AS and
AC samples. AgNP and CNF dispersions were mixed with a volume ratio of 1:1 for preparing the AgNP/CNF composite layer in the AM samples. $\mathrm{SiO}_{2}$ wafers or $\mathrm{SiO}_{2}$-fused glass slides (Silicon Materials, USA) of $20 \times 20 \mathrm{~mm}^{2}$ were used as substrates for AFM, GISAXS, GIWAXS, and contact-angle measurements. $\mathrm{SiO}_{2}$-fused glass slides were cut into $10 \times 10 \mathrm{~mm}^{2}$ as substrates for UV/vis spectrum measurements. All substrates were sonicated with acetone for $15 \mathrm{~min}$ and then sequentially cleaned with isopropanol and ultrapure water, followed by an acid bath $(87.5 \mathrm{~mL}$ of hydrogen peroxide $30 \%, 190$ $\mathrm{mL}$ of sulfuric acid $96 \%$, and $37.5 \mathrm{~mL}$ of ultrapure water) at $80{ }^{\circ} \mathrm{C}$ for $15 \mathrm{~min}$. All chemicals were purchased from Carl Roth $\mathrm{GmbH}$, Germany.

Spray-Coating. Layer-by-layer spray-coating at room temperature $\left(23^{\circ} \mathrm{C}\right)$ was performed by a spray-coating device (Compact JAU D555000, Spray Systems, Germany). CNF, AgNP, or their mixture solution was supplied by a siphon glass container connected to the spray device. Spray-coating was performed at a nitrogen gas pressure of $1 \mathrm{bar}$. The substrates were placed onto a heating plate of $120^{\circ} \mathrm{C}$. The distance between the nozzle and substrate was kept at $150 \mathrm{~mm}$. Three groups of samples were prepared as follows: AgNPs deposited on $\mathrm{SiO}_{2}$ wafers (AS), a AgNP/CNF LBL film with AgNPs coated on a CNF thin film predeposited on $\mathrm{SiO}_{2}$ wafers (AC), and a AgNP/CNF mixture deposited on $\mathrm{SiO}_{2}$ wafers (AM) (Figure 1). For CNF thin films with a thickness of $200 \mathrm{~nm}$, which was used as substrates for AgNP deposition in the AC samples, the deposition conditions were $0.2 \mathrm{~s}$ spraying and $10 \mathrm{~s}$ waiting alternated for 20 cycles. This is a wellestablished system for the investigation of the layering and selfassembly of MNPs on the MNP-CNF interface from our previous work. ${ }^{26}$ Therefore, the standard $\mathrm{CNF}$ and $\mathrm{SiO}_{2}$ substrates provide us the basis for studying the self-assembly of AgNPs during spray-coating by directly increasing the number of spray cycles. For the AgNP layer deposition in the AS and AC samples, the parameters were slightly changed into an alternated spraying of $0.1 \mathrm{~s}$ and waiting of $15 \mathrm{~s}$ with varying spray cycles from 1, 10, 50,100, to 150 . For the AgNP/CNF mixture in the AM samples, the protocols were $0.2 \mathrm{~s}$ spraying and $15 \mathrm{~s}$ waiting for 1, 10, 50, 100, and 150 cycles. The time for spray-coating in $\mathrm{AM}$ was doubled in comparison to AC and AS to ensure that the same amount of AgNPs was deposited at the same number of spray cycles among the three groups. The parameters for spray-coating are also optimized in an effort to prepare the AgNP layer with high homogeneity. ${ }^{21,30}$ We adopted the following name conventions for the three groups of samples: group name-number of spray cycles. For example, AC150 is the abbreviation of "AgNP solution deposited onto the CNF thin film for 150 spray cycles".

Atomic Force Microscopy (AFM). The AFM images were acquired with an NTEGRA probe Nano-Laboratory (NT-MDT, Russia) in the semicontact mode. ETALON cantilevers with a tip radius of $10 \mathrm{~nm}$ and a resonant frequency of $90 \mathrm{kHz}$ (NT-MDT, Russia) were used for all samples. For each sample, 20 pictures of $2 \mathrm{D}$ topography maps were taken with a scan size of $3 \times 3$ and $1 \times 1 \mu \mathrm{m}^{2}$. 

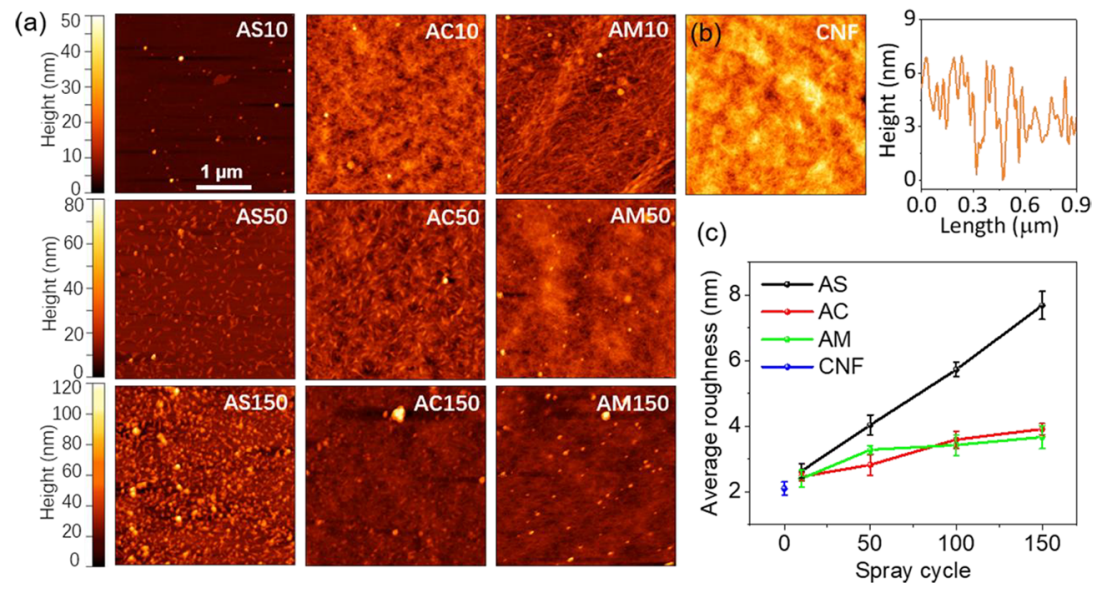

Figure 2. Morphological analysis of the spray-deposited AgNP layer. (a) AFM images of the AS, AC, and AM samples with 10 (top), 50 (middle), and 150 (bottom) spray cycles, respectively. (b) AFM image and the corresponding line cut of the CNF thin film. (c) Average roughness analysis of the AS, AC, and AM samples.

AFM data analysis, including line cuts, and average roughness were done by WSxM 5.0 software.

X-ray Scattering. The detailed morphology and crystallinity of the samples were investigated with GISAXS and GIWAXS, respectively. The X-ray scattering experiments were performed at the MiNaXS/P03 beamline of the PETRA III storage ring at DESY, Hamburg. ${ }^{31}$ The energy of X-ray was $12.85 \mathrm{keV}(\lambda=0.965 \AA)$ with a beam size of $80 \times 56 \mu \mathrm{m}^{2}$ (horizontal $\times$ vertical). One beam stop was used to shield the specular reflected beam. A sketch of the GISAXS and GIWAXS setup is shown in Figure S2, with $\alpha_{i}$ and $\alpha_{f}$ representing incident and exit angles, respectively. The sample-to-detector distance (SDD) was kept at $3040 \pm 2 \mathrm{~mm}$ in GISAXS and $115 \pm 1 \mathrm{~mm}$ in GIWAXS measurements. 2D GISAXS data were collected by a PILATUS 300k detector (Dectris Ltd., Switzerland) with a pixel size of $172 \times 172 \mu \mathrm{m}^{2}$, and GIWAXS data were collected by a Lambda $750 \mathrm{~K}$ detector (X-spectrum $\mathrm{GmbH}, \mathrm{Germany}$ ) with a pixel size of 55 $\times 55 \mu \mathrm{m}^{2}$. The incident angle $\alpha_{i}$ was set at $0.42^{\circ}$ for GISAXS and $0.11^{\circ}$ for GIWAXS measurements.

A lateral scanning with a step size of $100 \mu \mathrm{m}$ by moving the sample through the beam in the $y$-direction was performed to avoid X-ray beam-induced alteration of the samples. Each GISAXS pattern was acquired for $0.1 \mathrm{~s}$, and each GIWAXS pattern was acquired for $0.5 \mathrm{~s}$. 1D intensity distributions were extracted from 2D GISAXS data using the Directly Programmable Data Analysis Kit software (DPDAK) v1.4. $1^{32}$ and fitted by minimum chi-square estimation with cylindrical and spherical form factors including a structure factor as previously reported by Schaffer et al. ${ }^{33,34}$ For GIWAXS, the $q_{r}$ and $q_{z}$ plots were transformed by Grazing-incidence X-ray Scattering Graphical User Interface software (GIXSGUI) v1.7.1. ${ }^{35}$

UV/vis Spectroscopy. UV/vis transmission spectroscopy measurements were conducted on a custom build setup. For UV/vis spectra of the $\mathrm{AS}, \mathrm{AC}$, and $\mathrm{AM}$ samples, a $\mathrm{SiO}_{2}$-fused glass substrate (in the AS and AM samples) and a CNF thin film coated on a $\mathrm{SiO}_{2}-$ fused glass substrate (in the AC samples) were used as references. We used a spectrometer (OCEAN-FX-XR1-ES, Ocean Optics) covering a range of 200-1025 $\mathrm{nm}$ and a balanced deuterium-tungsten light source (DH-2000-BAL, Ocean Optics). Both light source and spectrometer were connected with solarized fibers and subsequently collimated with fused silica lenses (CVA100-COL, Thorlabs Inc.). The samples were mounted in the center between the collimation lenses, and the retrieved spectra were evaluated using the software package OceanView v1.6.5 (Ocean Optics). The transmittance spectra were created by measuring the background, substrate signal, and sample signal. Each integration was $4 \mathrm{~ms}$, and 10 scans were averaged for each spectrum. Optical spectra were smoothed by averaging 150 points using Origin2020 (OriginLab Corporation, Northampton, MA 01060, US).
Contact-Angle Measurement. Wettability measurements were facilitated by an OCA35 (Data Physics, Germany) contact-angle measuring system. An automatic syringe system was used to control the volume of deionized water droplet to $5 \mu \mathrm{L}$. At least five droplets were measured for each sample. Microscopic images were taken from each droplet, and the contact angle calculation was done by SCA20 software (Data Physics, Germany).

\section{RESULTS}

Surface Morphology. AgNPs deposited on $\mathrm{SiO}_{2}, \mathrm{AgNP} /$ CNF LBL film, and AgNP/CNF mixture deposited on $\mathrm{SiO}_{2}$ with $1,10,50,100$, and 150 spray cycles were prepared, respectively. AFM images of the AS, AC, and AM samples at 10, 50, and 150 spray cycles are shown in Figure 2a. An increase in the number of spray cycles leads to a higher $\mathrm{Ag}$ coverage $w$. The coverage $w$ follows in series: $w_{\mathrm{AS}}>w_{\mathrm{AC}}>w_{\mathrm{AM}}$. Densely packed CNFs are observed for all AC and AM samples. We can even distinguish a nonpercolated AgNP layer above the CNF layer in the samples AC50 and AC150. In agreement with our previous studies, ${ }^{26,27}$ no preferential orientation of cellulose nanofibrils or bundles is observed in the CNF thin film (Figure 2b). It is important to note that the AgNPs form laterally elongated agglomerates due to surface diffusion in the AS50 and AC50 samples (Figure 2a, middle). However, the AgNP agglomerates assemble into circular ones due to recoalescence in the AS150 and AC150 samples (Figure 2a, bottom).

Figure $2 b, c$ displays the surface roughness of the CNF layer and surface roughness values of the AS, AC, and AM samples as a function of the number of spray cycles, respectively. The roughness values are obtained using WsXM software v5.0 by estimating the root-mean-square value of the height distribution. ${ }^{36}$ The results suggest that the surface roughness of the nanoparticulate AS, AC, and AM films increases almost linearly with the amount of AgNP deposition. The variations in roughness at the same number of spray cycles may relate to different AgNP agglomeration patterns. Before 10 spray cycles, the roughness values of the AS1, AC1, and AM1 samples are $0.3 \pm 0.1,2.2 \pm 0.3$, and $2.1 \pm 0.3 \mathrm{~nm}$, which is mainly attributed to the difference of roughness between $\mathrm{SiO}_{2}$ wafer and CNF thin film. At 10 spray cycles, all samples show similar roughness around $2.6 \pm 0.3 \mathrm{~nm}$, signifying that the substrates become gradually covered by the deposited AgNP layer, which starts to dominate the surface morphology. From 10 to 150 

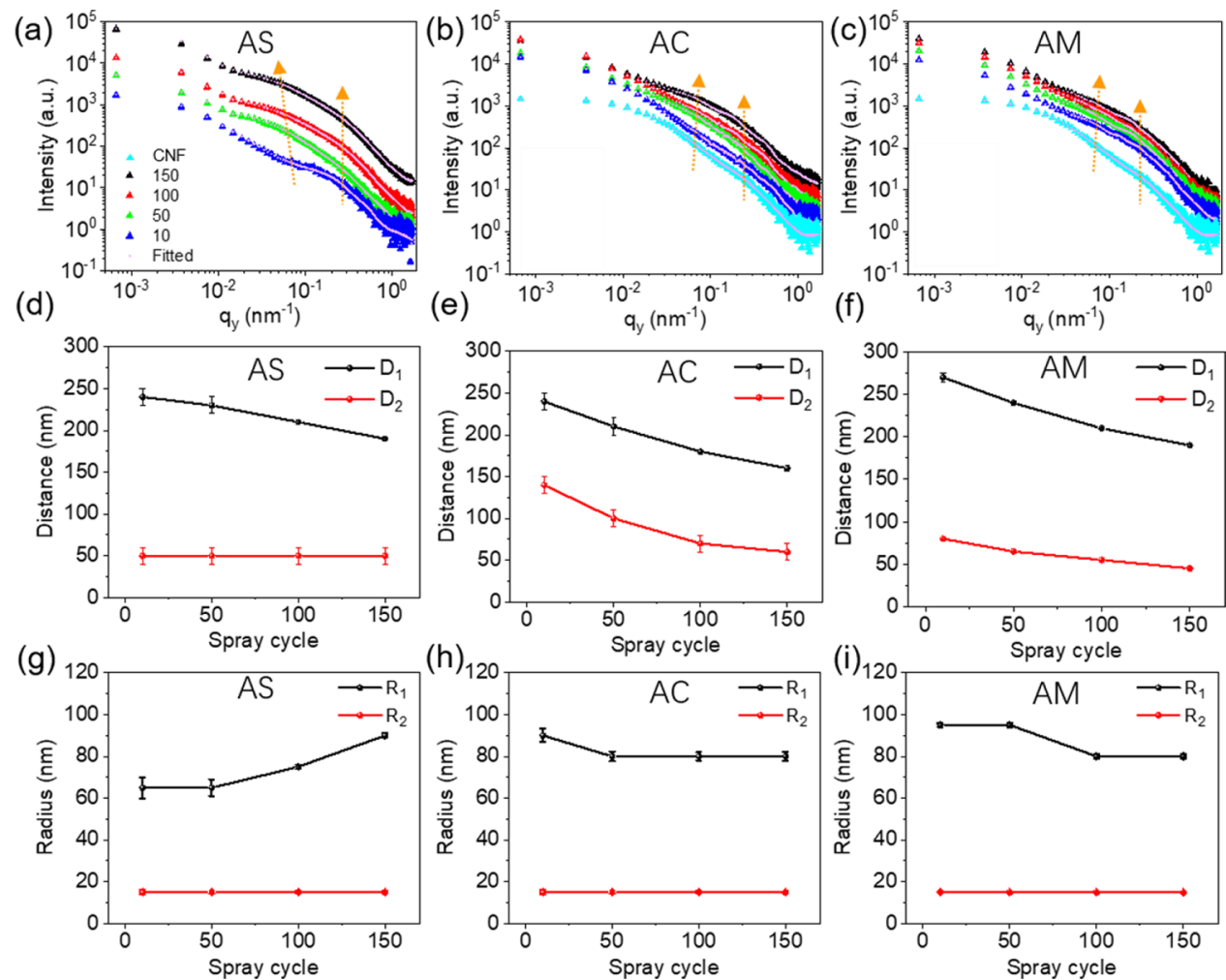

Figure 3. Horizontal line cuts of 2D GISAXS data of the (a) AS, (b) AC, and (c) AM samples at 10, 50, 100, and 150 spray cycles (details see Figure S5). The dashed lines present a fit to the data. The arrows guide to the eye showing the peak evolution as the number of spray cycles increases. (d)-(i) Extracted spherical structural parameters for AgNPs. For the AC and AM samples, which contain CNFs, the cylindrical structures for CNFs and spherical structures for AgNPs are both fitted, and for comparison, only the structures attributed to AgNPs are shown. (Cylindrical structures attributed to CNFs are shown in Figure S8.) (d)-(f) show the center-to-center distance (noted as "distance" for simplification) between adjacent spherical $A g$ nanostructures and $(g)-(i)$ show the radius of the $\operatorname{Ag}$ nanostructures, with $D_{1}, R_{1}, D_{2}, R_{2}$ corresponding to distance between and radius of AgNP agglomerates and individual AgNPs, respectively.

spray cycles, the average roughness values increase from $2.5 \pm$ 0.1 to $3.8 \pm 0.2 \mathrm{~nm}$ for the AC samples and $2.4 \pm 0.3$ to $3.6 \pm$ $0.4 \mathrm{~nm}$ in the AM samples, whereas it increases linearly from $2.6 \pm 0.3$ to $8.0 \pm 0.5 \mathrm{~nm}$ in the AS samples. This behavior is due to the fact that the CNF network favors a uniform AgNP assembly by sterically inhibiting AgNP agglomeration in the $\mathrm{AC}$ and $\mathrm{AM}$ samples, while the $\mathrm{SiO}_{2}$ substrate leads to a relatively nonrestricted agglomeration of AgNPs within 150 spray cycles. The slight roughness variations between the AC and AM samples are due to the higher extent of AgNP embedding in the AM samples compared to the AC samples at the same number of spray cycles. Thus, the roughness will be less affected by spray deposition in the case of the AM samples.

To investigate the influence of CNFs on the agglomeration of AgNPs, three line cuts with a length of $1 \mu \mathrm{m}$ are analyzed for each sample (Figure S3). Peaks of roughly $35 \pm 10 \mathrm{~nm}$ appear for all samples, which correspond to the diameter of an individual AgNP. Variations in height values above $40 \mathrm{~nm}$ suggest the existence of $\mathrm{Ag}$ nanostructures with different agglomeration sizes. Figure S3a shows that the heighst of the main peaks of the AS10, AS50, AS100, and AS150 samples are $28 \pm 3,33 \pm 6,48 \pm 11$, and $76 \pm 23 \mathrm{~nm}$, respectively. The peak height increases with increasing number of AgNP spray cycles, which might due to that the newly deposited AgNP predominantly anchor to the existing agglomerates and assemble into larger ones. ${ }^{20}$ Furthermore, the average peakto-peak distance drops from $350 \pm 270 \mathrm{~nm}$ in the AS10 sample to $172 \pm 130 \mathrm{~nm}$ in the AS150 sample. The increase in height and decrease in the peak-to-peak distance of AgNP agglomerates suggests a nonrestrictive agglomeration pattern of AgNPs in the AS samples. ${ }^{37}$ In the AC samples, both AgNP agglomerates and individual AgNPs are well distributed on the CNF layer (Figure 2a, middle). As the number of spray cycles increased from 10 to 150 , we observe an increase in the peak height from $30 \pm 9$ to $62.3 \pm 0.4 \mathrm{~nm}$ and a relatively constant peak width of $130 \pm 25 \mathrm{~nm}$ (Figure S3b). An explanation for these changes is that the newly deposited AgNP solution partially dissolves the previously formed AgNP agglomerates, and the detached AgNPs penetrate into the CNF substrate ${ }^{38,39}$ in the AC samples, while the dissolved agglomerates recoalesce and reassemble on the $\mathrm{SiO}_{2}$ substrate in the AS samples. The agglomeration pattern of AgNPs in the AM samples is similar to the AC samples (Figure S3c). The height of AgNP agglomerates slightly increased from $36 \pm 4$ to $48 \pm 19 \mathrm{~nm}$, and the peak-to-peak distance drops from $256 \pm 43$ to $236 \pm$ $164 \mathrm{~nm}$ as the number of spray cycles increases from 10 to 150. The lower height values in the AM samples compared to the AC samples can be attributed to the partial embedding of AgNP agglomerates within the CNF network. The larger distance in the AM samples in comparison to the AC samples is due to the $3 \mathrm{D}$ distribution of $\mathrm{Ag}$ nanostructures (including AgNP agglomerates and individual AgNPs) through the CNF network in the AM samples instead of $2 \mathrm{D}$ distribution in the AC samples. ${ }^{40}$ The diverse morphologies of Ag nanostructures among the AS, AC, and AM samples suggest the existence of different patterns of AgNP self-assembly on smooth $\mathrm{SiO}_{2}$ 
substrates and on/within the CNF thin film. CNFs not only provide a network structure to restrict AgNP agglomeration but also promote partial dissolution of AgNP agglomerates to form a smoother surface.

Layer Morphology. To characterize the layer morphology and self-assembly of AgNPs at the nanoscale, GISAXS measurements are performed for the spray-deposited AS, AC, and AM samples at 10,50,100, and 150 spray cycles. 2D GISAXS data are shown in Figure S4. Vertical line cuts (offcentered cuts) along the $q_{z}$ direction at an offset of $0.03 \mathrm{~nm}^{-1}$, and horizontal line cuts along the $q_{y}$ direction at the Yoneda peak position ${ }^{41}$ were performed (Figure S5) to extract the structural information perpendicular and parallel to the surface, respectively.

The comparison of vertical off-centered line cuts of the AS, $\mathrm{AC}$, and $\mathrm{AM}$ samples at different spray cycles is shown in Figure S6. Qualitatively, the larger peak intensity of the AC10 than the AS10 sample is related to a higher background roughness of CNF than the AgNP layer at the early stage of spray-coating. ${ }^{24}$ However, after 50 spray cycles, the roughness of the AC samples becomes lower than that of the AS samples, which may suggest a lower agglomeration rate of AgNPs on CNF substrates than $\mathrm{SiO}_{2}$ substrates. Another important factor is the partial embedding of AgNP agglomerates into the CNF layer, which is consistent with the analysis from AFM measurements. The peak intensities from 10 to 150 spray cycles are nearly consistent for the AC and AM samples. The similar trends of roughness evolution between the AM and AC samples can be rationalized by the important role of the network structure of CNFs in AgNP agglomeration. The relatively stable intensities in the AM samples indicate both the restricted agglomeration of AgNPs by the surrounding CNF matrix and a constant roughness of the spray-coated CNFs at different deposited thickness. The higher peak intensity of the AM100 than the AC100 sample is due to the lower content of $\mathrm{CNF}$ in the AM samples than in the AC samples. Therefore, AgNP agglomerates could not be fully covered by the CNF matrix. The lower roughness of the AM150 than the AC150 samples may attribute to the smaller size of AgNP agglomerates, or more probably the higher embedding rate of AgNP agglomerates in CNFs within the AM samples.

In horizontal direction, two broad smeared peaks are found in the AS, AC, and AM samples, indicating the presence of two $\mathrm{Ag}$ nanostructures with different sizes (Figure $3 \mathrm{a}-\mathrm{c}$ ). In the AS samples, the peak at $q_{y} \approx 0.04 \mathrm{~nm}^{-1}$ increases and shifts toward lower values as deposition proceeds, and this is related to the size increase of a large nanostructure. We deduce that this signal originates from AgNP agglomerates. However, the peak at higher $q_{y}$ values $\left(q_{y} \approx 0.21 \mathrm{~nm}^{-1}\right)$ remains nearly unchanged in position and becomes stronger with increasing number of spray cycles. This feature corresponds to the center-to-center distance between individual AgNPs. With increasing AgNP deposition in the AC samples, the scattering intensity increases primarily at higher $q_{y}$ values in which the amplitude increase at $q_{y} \approx 0.21 \mathrm{~nm}^{-1}$ implies that individual AgNPs become closer in distance (Figure $3 \mathrm{~b}$ ). The peak at $q_{y} \approx 0.04 \mathrm{~nm}^{-1}$ slightly enhances and shifts to higher $q_{y}$ values, suggesting that AgNP agglomerates become closer in distance and slightly decrease in radius (as revealed by the GISAXS fits in the next part, see Figure $3 \mathrm{e}, \mathrm{h}$ ). It is important to note that, different from AFM measurements, where a higher AgNP coverage leads to a weaker CNF signal, GISAXS is a probe that monitors the morphology both, in parallel and vertical directions. Therefore, the intensity of the CNF peaks almost does not change in the AC samples with increasing number of spray cycles as the Xray beam penetrates the full composite film. Similar to the AS and AC samples, two peaks at $q_{y} \approx 0.21 \mathrm{~nm}^{-1}$ and $q_{y} \approx 0.04$ $\mathrm{nm}^{-1}$ exist in the AM samples, corresponding to individual AgNPs and AgNP agglomerates, respectively (Figure 3c). Different from the AC samples, only a slight increase in the intensity of both the two peaks with a slight shift to lower $q_{y}$ values is observed in the AM samples, indicating that the radius of AgNP agglomerates and individual AgNPs remain nearly constant regardless of the number of spray cycles and the simultaneous existence of an increasing amount of large and small structures from both AgNPs and CNFs in the AM samples.

To analyze the GISAXS data based on real-space morphological features, we fit the horizontal line cuts with a model assuming cylindrical shapes for CNF-related nanostructures $^{26}$ and spherical shapes for AgNP-related nanostructures. $^{33}$ The used GISAXS fit model has been proven by literature. ${ }^{42-44}$ The radius $R$ and center-to-center distance $D$ of these model shapes (cylinders and spheres) were varied in the fit. This model enables us to directly compare the parameters of the fitted nanostructures between GISAXS and AFM measurements. Table $S 1$ introduces the parameters involved in the GISAXS fit. Figure $3 d-i$ shows the fitted $R$ and $D$ values of the AS, AC, and AM samples. In the AS10 sample, AgNP agglomerates with $R_{1}=65 \pm 5 \mathrm{~nm}$ in a distance of $D_{1}=240 \pm$ $10 \mathrm{~nm}$ and individual AgNPs of $R_{2}=15.0 \pm 1.0 \mathrm{~nm}$ in a distance of $D_{2}=50 \pm 10 \mathrm{~nm}$ are found. In the AS150 sample, the agglomerates show $R_{1}=90.0 \pm 1.0 \mathrm{~nm}$ in a distance of $D_{1}$ $=190.0 \pm 1.0 \mathrm{~nm}$, and individual AgNPs show $R_{2}=15.0 \pm 0.5$ $\mathrm{nm}$ in a distance of $D_{2}=50 \pm 10 \mathrm{~nm}$ (Figure $3 \mathrm{~d}, \mathrm{~g}$ ). Both the amplitude of AgNP agglomerates and individual AgNPs increase with continuous deposition (Figure S7), indicating that individual AgNPs increasingly contribute to the selfassembly of AgNP agglomerates, and existing AgNP agglomerates become the nuclei for adsorption of newly deposited AgNPs as spray-coating proceeds in the AS samples. Due to the complex system of AgNP-CNF nanocomposites, we fitted the CNF network before fitting AgNPs in AC and AM samples. Three cylindrical structures are identified in $\mathrm{CNF}$ thin films, with $R c_{1}=30 \pm 2 \mathrm{~nm}, R c_{2}=11 \pm 3 \mathrm{~nm}$, and $R c_{3}=$ $2.1 \pm 1.0 \mathrm{~nm}$ and $D c_{1}=73.0 \pm 1.0 \mathrm{~nm}, D c_{2}=75.0 \pm 1.0 \mathrm{~nm}$, and $D c_{3}=10 \pm 3 \mathrm{~nm}$ (Figure S8), respectively, which agrees well with our previous results. ${ }^{26}$ This enables us to extract the $\mathrm{Ag}$ nanostructures on and within the CNF network. Based on these parameters and keeping them constant, we added the AgNP fits to retrieve their radii and distances. This reduces the number of free parameters and increases reliability of the fit. In case of the AC samples, the radius of AgNP agglomerates $\left(R_{1}\right)$ slightly decreases from $90 \pm 3$ to $80 \pm 2 \mathrm{~nm}$, which demonstrates the deduced effect of partial dissolution of AgNP agglomerates from AFM measurements. The smaller radius of AgNP agglomerates in the AC than the AS samples may due to the hindering effect of the network structure of CNFs on coalescence of individual AgNPs (Figure 3e,h). Similarly, AgNP agglomerates in the AM10 sample show $R_{1}=95.0 \pm 1.0$ $\mathrm{nm}$ and $D_{1}=270 \pm 5 \mathrm{~nm}$, while in the AM150 sample, AgNP agglomerates display a radius of $R_{1}=80.0 \pm 1.0 \mathrm{~nm}$ with a distance of $D_{1}=180.0 \pm 1.0 \mathrm{~nm}$ (Figure 3f,i). Since $\mathrm{Ag}$ nanostructures are mainly distributed at the AgNP-CNF interface in the AC samples where partial dissolution of AgNP agglomerates primarily occurs, the dissolution effect is stronger 
in the $\mathrm{AC}$ than in the $\mathrm{AM}$ samples, where $\mathrm{Ag}$ nanostructures are distributed through the thickness direction of the film. Therefore, the radius of AgNP agglomerates is slightly higher in the AM than in the AC samples. The distance between individual AgNPs decreases as spray-coating proceeds in the $\mathrm{AC}$ and $\mathrm{AM}$ samples, indicating a more homogeneous distribution of AgNPs on/within CNFs than on $\mathrm{SiO}_{2}$ substrates. The GISAXS fit results generally corroborate the deduced morphology of Ag nanostructures from the AFM analysis.

Crystallinity Analysis. GIWAXS measurements are conducted to investigate the influence of the CNF network on the crystallinity of the AgNPs. Differences in the scattering patterns and relative intensities are observed among the AS, AC, and AM samples at the same number of spray cycles (Figure 4a and Figure S9a,b). Generally, two scattering peaks
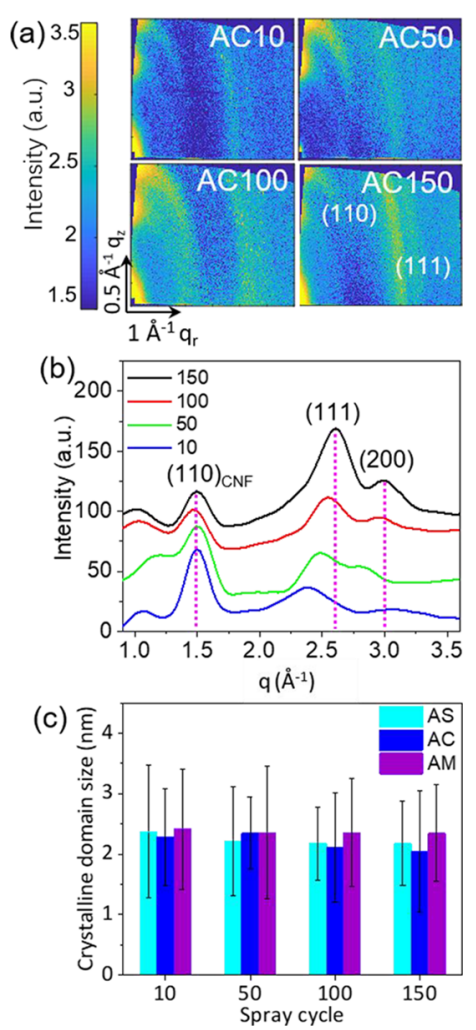

Figure 4. (a) 2D GIWAXS data $\left(q_{r}, q_{z}\right.$ maps) of the AgNP layer in the AC samples for $10,50,100$, and 150 spray cycles. (b) $q$ plot from the radial integration of the reshaped $q_{\mathrm{r}}, q_{z}$ map (see Figure S10, 11 for details) of the AC samples. (c) Minimum crystallite sizes of AgNPs calculated from the FHWM of the (111) peak in the AS, AC, and AM samples.

are observed at around $q=2.6 \AA^{-1}$ and $q=3.1 \AA^{-1}$, corresponding to (111) and (200) planes of the face centered cubic (fcc) structure of AgNP, respectively. ${ }^{45,46} q$ represents the total wave vector containing the in-plane $q_{x}, q_{y}$ component and out-of-plane $q_{z}$ component.

To probe the evolution of the crystallinity of AgNPs during spray-coating, the integrated intensity of the peaks as a function of $q$ is extracted from the 2D GIWAXS profiles by radial integrations (Figure $S 10)$. Two scattering peaks around $q=2.6 \AA^{-1}$ and $q=3.1 \AA^{-1}$ (Figure $4 \mathrm{~b}$ and Figure S9c,d) confirm the existence of the (111) and (200) planes of $\mathrm{Ag}$ nanocrystallites. For the AS, AC, and AM samples, the scattering intensity from the (111) planes is stronger than that of the (200) planes; therefore, only the (111) signal $(q=2.6$ $\left.\AA^{-1}\right)$ is used in the following crystallinity analysis. The relative intensity of the (111) scattering peak increases with continuous spray-deposition in the AS, AC, and AM samples due to material gain (Figure $4 \mathrm{~b}$, Figure $59 \mathrm{c}, \mathrm{d}$ ). In addition to the scattering peaks of AgNPs, the peak at $q=1.4 \AA^{-1}$ in the $\mathrm{AC}$ and $\mathrm{AM}$ samples is identified, corresponding to the (110) scattering plane of the CNFs. As expected, the intensity of the (110) scattering peak of CNFs remains constant as the number of AgNP spray cycle increases in the AC samples, while it becomes stronger as the number of AgNP/CNF spray cycle increases in the $\mathrm{AM}$ samples, corresponding to the differential amount of CNFs between the AC and AM samples at the same number of spray cycles.

To further investigate the evolution of the (111) scattering patterns of AgNPs, a Gaussian curve is fitted to the $q$ plot in the range from $2.6<q<3.1 \AA^{-1}$ (Figure S11). The minimum crystalline size of AgNP is estimated from the full width at half maximum (FWHM) of the fitted (111) scattering peak deducing the instrumental resolution measured from $\mathrm{LaB}_{6}$, via the adapted Debye-Scherrer formula for grazing-incidence scattering ${ }^{47,48}$ with a K-factor of 0.94 . Within the experimental error, the minimum crystalline size of AgNPs remains constant around $2.4 \pm 0.9 \mathrm{~nm}$ regardless of the increasing deposition amount of AgNPs or AgNP/CNF mixture (Figure 4c). Our results demonstrate that the morphology of the substrates and the amount of AgNPs or the AgNP/CNF mixture deposited have no impact on the crystallite size of AgNPs. This enables us to fabricate a homogenous AgNP/CNF film with a stable crystallinity by repetitive spray-coating.

Self-Assembly Model. Based on the comprehensive analyses of AFM, GISAXS and GIWAXS results, we summarize the morphologies of AgNPs at multiple scales (Figure 5a), the schematic structure of AgNP-CNF composites (Figure 5b), and identify three distinct self-assembly models of AgNPs in the AS, AC, and AM samples (Figure 5c). Random agglomeration predominates in the self-assembling process of AgNPs on the $\mathrm{SiO}_{2}$ substrate. With continuous deposition, the AgNP agglomerates become larger in size and closer in distance. Therefore, the surface coverage increases, and the AgNP layer is coarsening. For the AC samples, the AgNP agglomerates are partially embedded into CNFs in the early stages of the deposition (as revealed by the distance between CNFs and the radius of AgNPs from the GISAXS fit) and begin to assemble on top of the CNF layer as deposition proceeds. This finding can be rationalized from the AFM analysis. It is observed that a small amount of AgNP agglomerates is inserted to the CNF layer, and the average distance between CNFs deduced from AFM (about $75 \mathrm{~nm}$ ) is higher than the diameter of the individual AgNPs deduced from the GISAXS fit (about $30 \mathrm{~nm}$ ). In the horizontal direction, the radius of AgNP agglomerates gradually decreases when spray-coating continues via partial dissolution, indicating the crucial role of the substrate morphology on the selfassembly of the AgNPs (Figure 5c, left, middle). In the case of the AM samples, CNFs self-assemble simultaneously with the AgNPs due to the coexistence of van der Waals force between $\mathrm{Ag}-\mathrm{Ag}$ and electrostatic $\mathrm{Ag}-\mathrm{CNF}$ interactions (Figure 5b,c, right). Similar to the AC samples, agglomeration of the AgNPs is also affected by a combination of a spatial hindering effect and stronger partial dissolution effect of the CNF matrix than in the AC samples. 


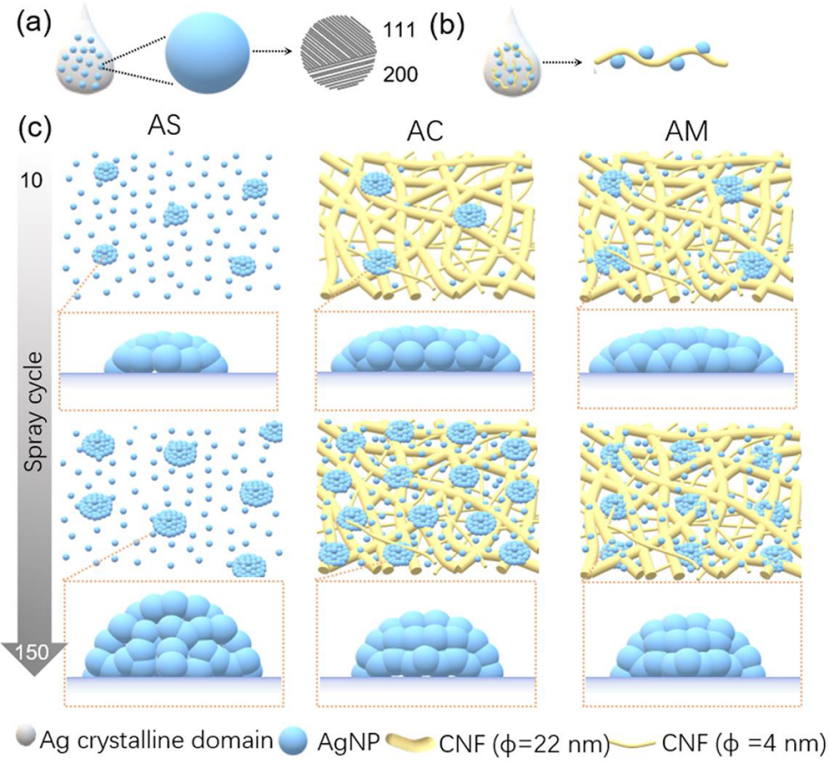

Figure 5. Schematic diagram showing the self-assembly of spraycoated AgNPs in the AS, AC, and AM samples. (a) Deduced Ag morphologies from macroscale to nanoscale. $\Phi=2 \times R$ is used as the diameter of the nanostructures in CNFs for comparison with the center-to-center distance. (b) Sketch of the morphology of AgNPCNF composites. (c) Self-assembly of AgNPs in horizontal and perpendicular directions with increasing number of spray cycles from top and side views.

Applications. To investigate the wettability of the AgNPcontaining layer after spray-coating, contact-angle measurements of the AS, AC, and AM samples at different spray cycles are performed at room temperature. The droplet morphologies are shown in Figure S12. The contact angles of the AS10, AC10, and AM10 samples are $50^{\circ} \pm 2^{\circ}, 38.0^{\circ} \pm 1.0^{\circ}$, and $45.0^{\circ} \pm 1.0^{\circ}$, indicating a higher wettability of the CNF thin film compared to the $\mathrm{SiO}_{2}$ substrate (Figure 6a). With ongoing
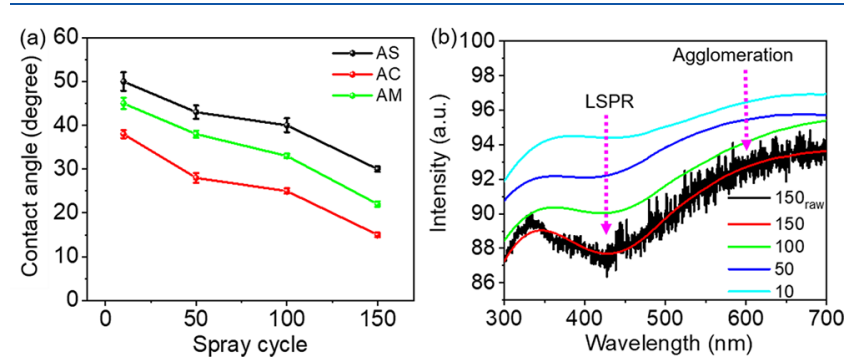

Figure 6. Applications of AgNP/CNF films. (a) Water contact angles of the AC, AS, and AM samples as a function of number of spray cycles. (b) UV/vis spectra of the AC (AgNP/CNF LBL films) samples from 10 to 150 spray cycles. The black line shows the exemplary raw spectra for the AC150 sample.

deposition, the contact angle in all three groups decreases. This suggests that AgNP deposition improves the surface hydrophilicity. The hydrophilicity change is induced by the adding of AgNP. The change in hydrophilicity is larger in combination with CNF. The AM and AC samples show a similar wettability evolution as spray-coating proceeds. We propose that surface roughness plays an important role in the wetting behavior of the AgNP layer. The lower contact angles of AC150, AM150 than AS150 suggest that the use of CNF substrate leads to a more hydrophilic surface. ${ }^{49}$ Furthermore, the water contact angles of AC and AM samples measured after three months of storing in ambient conditions are almost the same with that measured immediately after synthesis, suggesting an excellent stability of the AgNP/CNF films. Tailorable wettability ${ }^{26,50}$ is crucial for the functionalization of thin films with antifouling, ${ }^{51}$ detergent-free cleaning, ${ }^{52}$ or antifogging ${ }^{53}$ properties in biomedical and industrial applications.

$\mathrm{UV} /$ vis spectra of the AS, AC, and AM samples at 10, 50, 100 , and 150 spray cycles are examined to study the influence of CNFs on the plasmonic properties of the AgNP layer. The transmission spectrum is recorded over a wavelength range from 200 to $1025 \mathrm{~nm}$. Figure $6 \mathrm{~b}$ and Figure S13 show the comparison of the relative transmissions of the AC, AS, and AM samples at different spray cycles. The spectra are smoothed to show the features of the LSPR peak of AgNP agglomeration. The LSPR peak of AgNP emerges at around 450 to $500 \mathrm{~nm},{ }^{54-58}$ which could be influenced by a combined effect from the surrounding medium, size, shape, and interparticle distance of $\mathrm{Ag}$ nanostructures. Therefore, we attribute the dip in the transmission UV/vis spectrum to the LSPR peak of AgNPs. ${ }^{59,60}$ The spectrum from 500 to $700 \mathrm{~nm}$ following the LSPR peak may originate from AgNP agglomerates. For the AS samples, the LSPR peak remains almost unchanged in the position when the spray cycle increased from 10 to 150 . For the $\mathrm{AC}$ and $\mathrm{AM}$ samples, the LSPR peak is slightly blue-shifted from 443 to $424 \mathrm{~nm}$ and 462 to $436 \mathrm{~nm}$, respectively, and the transmission decreases with the number of spray cycles, which could be related to the decrease in the center-to-center distance of the AgNP agglomerates or a variation in the dielectric environment. Based on these results, we propose that the CNF network contributes to a higher LSPR intensity in the AC and AM samples by providing a uniform distribution of the AgNPs with a smaller agglomerate size and interparticle distance at the nanoscale, which is promising for optical and catalytic applications. ${ }^{61-64}$

The novelty of this work is two-fold; on the one hand, we use CNF both as a substrate and structural framework for AgNP deposition. This demonstrates the potential of CNF for versatile, tailorable, and homogeneous nanoparticle layer fabrication on the nanoscale. On the other hand, we obtained the AgNP/CNF film not only with layer-by-layer spraycoating $^{65}$ (AC samples) but also by a one-step spray-coating process (AM samples), resulting in AgNP/CNF nanocomposite films with similar morphological, wetting, and plasmonic properties. The mechanism of AgNP/CNF with higher hydrophilicity and plasmonic properties is due to a more homogeneous distribution of AgNPs with specific morphologies provided by the CNF network. This contributes to a system for fabrication of AgNP layers with defined nanostructures for tailorable optical, catalytic, and other applications. The nanostructures of the AgNP layer play an important role in the applications of AgNP-based thin films. Essential structural parameters of AgNPs include the variations in the height of the AgNP layer, the center-to-center distance between neighboring Ag nanostructures, and the radius of $\mathrm{Ag}$ nanostructures. Surface roughness, which correlates to the height variations of AgNP agglomerates in the perpendicular direction, is crucial for the wetting behavior of the AgNP layer. Therefore, AgNPs spray-coated onto (the AC samples) or within (the AM samples) the CNF thin film exhibit higher hydrophilicity than AgNPs sprayed on the smooth $\mathrm{SiO}_{2}$ 
substrate. Furthermore, a homogeneous AgNP layer will give excellent plasmonic properties, which could be applied for label-free chemical sensing ${ }^{66}$ and wearable SERS sensors. ${ }^{67}$ Therefore, the use of CNFs provides a scaffold for the AgNP deposition, leading to a homogeneous AgNP/CNF composite film with higher LSPR intensity compared to the $\mathrm{AgNP} / \mathrm{SiO}_{2}$ film. Another potential application of the fabricated AgNP/ CNF films is the antibacterial textiles. ${ }^{28,65}$

\section{CONCLUSIONS}

We have developed a scalable approach to assemble highly homogeneous AgNP films by spray-coating of AgNPs onto and within the CNF network. Individual AgNPs are composed of multiple crystalline domains, and the size the crystalline domain is unaffected by the substrate morphology and spraycoating process. In the lateral direction, the combined influence of partial dissolution and hindering effects from the CNF matrix lead to a decrease in the radius and center-tocenter distance between the AgNP agglomerates in the AgNP/ $\mathrm{CNF}$ films. Only a slight increase in surface roughness of the AgNP/CNF films is observed during spray-coating. Three selfassembly models for (1) AgNPs deposited on $\mathrm{SiO}_{2}$ substrate, (2) AgNPs deposited on CNF layer, and (3) the mixture of AgNP/CNF deposited on $\mathrm{SiO}_{2}$ substrates are developed from the comprehensive structure analysis. Our study proposes a detailed understanding on the self-assembly of AgNPs on/ within CNFs and provides a novel route to obtain homogeneous MNP distributions using CNFs as a universal substrate or matrix. The smooth and uniform distribution of AgNPs lead to a high hydrophilicity and LSPR intensity in the AgNP/CNF composite films, which shows potential applications as smart sensors for detection of biomacromolecules with tailorable roughness, morphology, and wettability.

\section{ASSOCIATED CONTENT}

\section{(s) Supporting Information}

The Supporting Information is available free of charge at https://pubs.acs.org/doi/10.1021/acsanm.0c02819.

Sketch of the scattering geometry of GISAXS and GIWAXS; AFM height analysis; 2D GISAXS data; GISAXS line cuts region, GISAXS off-center vertical line cuts, amplitude evolution deduced from GISAXS fits, and GISAXS fits of CNF; GISAXS fit parameters; GIWAXS data of the AS and AM samples; GIWAXS radial-integration region; water contact-angle droplet morphology, UV/vis spectra of AS, AC, and AM samples, and additional notes on sample preparation, AFM analysis, and GISAXS (PDF)

\section{AUTHOR INFORMATION}

\section{Corresponding Authors}

Qing Chen - School of Chemistry and Material Science, University of Science and Technology of China, 230026 Hefei, China; Deutsches Elektronen-Synchrotron, 22607 Hamburg, Germany; 이이.org/0000-0002-1828-7797; Email: qing.chen@desy.de

Stephan V. Roth - Deutsches Elektronen-Synchrotron, 22607 Hamburg, Germany; Fibre and Polymer Technology, KTH Royal Institute of Technology, 10044 Stockholm, Sweden; ○ orcid.org/0000-0002-6940-6012; Email: svroth@kth.se

\section{Authors}

Calvin J. Brett - Deutsches Elektronen-Synchrotron, 22607 Hamburg, Germany; Department of Engineering Mechanics and Wallenberg Wood Science Center, KTH Royal Institute of Technology, 10044 Stockholm, Sweden; (1) orcid.org/ 0000-0001-5789-6299

Andrei Chumakov - Deutsches Elektronen-Synchrotron, 22607 Hamburg, Germany; 이이.org/0000-0003-31959356

Marc Gensch - Deutsches Elektronen-Synchrotron, 22607 Hamburg, Germany; Lehrstuhl für Funktionelle Materialien, Physik Department, Technische Universität München, 85748 Garching, Germany; (1) orcid.org/0000-0001-5514-8158

Matthias Schwartzkopf - Deutsches Elektronen-Synchrotron, 22607 Hamburg, Germany; (1) orcid.org/0000-0002-21159286

Volker Körstgens - Lehrstuhl für Funktionelle Materialien, Physik Department, Technische Universität München, 85748 Garching, Germany; (1) orcid.org/0000-0001-7178-5130

L. Daniel Söderberg - Department of Engineering Mechanics and Wallenberg Wood Science Center, KTH Royal Institute of Technology, 10044 Stockholm, Sweden; (1) orcid.org/ 0000-0003-3737-0091

Anton Plech - Institute for Photon Science and Synchrotron Radiation, Karlsruhe Institute of Technology (KIT), 76021 Karlsruhe, Germany

Peng Zhang - School of Material Science and Engineering, PCFM lab, Sun Yat-sen University, 510275 Guangzhou,

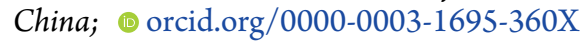

Peter Müller-Buschbaum - Lehrstuhl für Funktionelle Materialien, Physik Department and Heinz Maier Leibniz Zentrum (MLZ), Technische Universität München, 85748 Garching, Germany; 이이이.org/0000-0002-9566-6088

Complete contact information is available at: https://pubs.acs.org/10.1021/acsanm.0c02819

\section{Author Contributions}

The research has been designed by S.V.R., P.Z., and D.S. CNF and AgNP aqueous dispersions are provided by D.S. and A.P., respectively. Q.C., C.J.B., A.C., M.G., and M.S. conducted AFM, GISAXS, GIWAXS, and contact-angle measurements. C.J.B. conducted UV/vis measurements. Q.C., S.V.R., M.S., V.K., and P.M.B. performed data analysis with support and recommendations from all co-authors. The manuscript was written with contributions from all authors.

\section{Funding}

This work is supported by the Helmholtz-OCPC program (no. 20191004). Q.C. and S.V.R. acknowledge the kind financial support from the DESY strategic fund (DSF) "Investigation of processes for spraying and spray-coating of hybrid cellulosebased nanostructures". V.K. and P.M.B. acknowledge funding from TUM.solar in the context of the Bavarian Collaborative Research Project Solar Technologies Go Hybrid (SolTech), the Deutsche Forschungsgemeinschaft (DFG, German Research Foundation) under Germany's Excellence Strategy-EXC 2089/1-390776260 (e-conversion) and the International Research Training Group 2022 Alberta/Technical University of Munich International Graduate School for Environmentally Responsible Functional Hybrid Materials (ATUMS). P.Z. acknowledges funding from National Natural Science Foundation of China (no. 11905306) and Fundamental Research Funds for the Central Universities (no. 19lgpy14). 


\section{Notes}

The authors declare no competing financial interest.

\section{ACKNOWLEDGMENTS}

The authors thank the synchrotron light source PETRA III and beamline P03 at Deutsches Elektronen-Synchrotron (DESY) for beam time allocation.

\section{ABBREVIATIONS}

AS, AgNP deposited on $\mathrm{SiO}_{2}$ AC, AgNP/CNF LBL film

$\mathrm{AM}, \mathrm{AgNP} / \mathrm{CNF}$ mixture deposited on $\mathrm{SiO}_{2}$ MNP, metallic nanoparticle

AFM, atomic force microscopy

AgNP, silver nanoparticle

CNF, cellulose nanofibril

DANFC, dialdehyde nanofibrillated cellulose

DPDAK, directly programmable data analysis kit

fcc, face centered cubic

GISAXS, grazing-incidence small-angle X-ray scattering

GIWAXS, grazing-incidence wide-angle X-ray scattering

GIXSGUI, grazing-incidence X-ray scattering graphical user interface

LBL, layer-by-layer

LSPR, localized surface plasmon resonance

SDD, sample-to-detector distance.

\section{REFERENCES}

(1) Liu, X. H.; Ma, J. G.; Niu, Z.; Yang, G. M.; Cheng, P. An Efficient Nanoscale Heterogeneous Catalyst for the Capture and Conversion of Carbon Dioxide at Ambient Pressure. Angew. Chem., Int. Ed. 2015, 54, 988-991.

(2) Xie, Y.; Yan, B.; Xu, H.; Chen, J.; Liu, Q.; Deng, Y.; Zeng, H. Highly Regenerable Mussel-Inspired $\mathrm{Fe}_{3} \mathrm{O}_{4} @$ Polydopamine-Ag CoreChell Microspheres as Catalyst and Adsorbent for Methylene Blue Removal. ACS Appl. Mater. Interfaces 2014, 6, 8845-8852.

(3) Wu, M.; Ma, B.; Pan, T.; Chen, S.; Sun, J. Silver-NanoparticleColored Cotton Fabrics with Tunable Colors and Durable Antibacterial and Self-Healing Superhydrophobic Properties. Adv. Funct. Mater. 2016, 26, 569-576.

(4) Chernousova, S.; Epple, M. Silver as Antibacterial Agent: Ion, Nanoparticle, and Metal. Angew. Chem., Int. Ed. 2013, 52, 1636-1653.

(5) Li, S.; Xu, L.; Sun, M.; Wu, X.; Liu, L.; Kuang, H.; Xu, C. Hybrid Nanoparticle Pyramids for Intracellular Dual MicroRNAs Biosensing and Bioimaging. Adv. Mater. 2017, 29, 1606086.

(6) Huang, J.; Li, D.; Zhao, M.; Mensah, A.; Lv, P.; Tian, X.; Huang, F.; Ke, H.; Wei, Q. Highly Sensitive and Stretchable CNT-Bridged AgNP Strain Sensor Based on TPU Electrospun Membrane for Human Motion Detection. Adv. Electron. Mater. 2019, 5, 1900241.

(7) Matsuhisa, N.; Inoue, D.; Zalar, P.; Jin, H.; Matsuba, Y.; Itoh, A.; Yokota, T.; Hashizume, D.; Someya, T. Printable Elastic Conductors by In Situ Formation of Silver Nanoparticles from Silver Flakes. Nat. Mater. 2017, 16, 834-840.

(8) Park, M.; Im, J.; Shin, M.; Min, Y.; Park, J.; Cho, H.; Park, S.; Shim, M. B.; Jeon, S.; Chung, D. Y.; Bae, J.; Park, J.; Jeong, U.; Kim, K. Highly Stretchable Electric Circuits from a Composite Material of Silver Nanoparticles and Elastomeric Fibres. Nat. Nanotechnol. 2012, 7, 803-809.

(9) Sun, Y.; Xia, Y. Shape-Controlled Synthesis of Gold and Silver Nanoparticles. Science 2002, 298, 2176-2179.

(10) Zhang, Q.; Ge, J.; Pham, T.; Goebl, J.; Hu, Y.; Lu, Z.; Yin, Y. Reconstruction of Silver Nanoplates by UV Irradiation: Tailored Optical Properties and Enhanced Stability. Angew. Chem., Int. Ed. 2009, 48, 3516-3519.
(11) Wiley, B.; Sun, Y.; Xia, Y. Synthesis of Silver Nanostructures with Controlled Shapes and Properties. Acc. Chem. Res. 2007, 40, 1067-1076.

(12) Yu, W. W.; White, I. M. Inkjet Printed Surface Enhanced Raman Spectroscopy Array on Cellulose Paper. Anal. Chem. 2010, 82, 9626-9630.

(13) Hu, L.; Zheng, G.; Yao, J.; Liu, N.; Weil, B.; Eskilsson, M.; Karabulut, E.; Ruan, Z.; Fan, S.; Bloking, J. T.; McGehee, M. D.; Wågberg, L.; Cui, Y. Transparent and Conductive Paper from Nanocellulose Fibers. Energy Environ. Sci. 2013, 6, 513-518.

(14) Kaushik, M.; Moores, A. Review: Nanocelluloses as Versatile Supports for Metal Nanoparticles and Their Applications in Catalysis. Green Chem. 2016, 18, 622-637.

(15) Toncheva, A.; Khelifa, F.; Paint, Y.; Voué, M.; Lambert, P.; Dubois, P.; Raquez, J.-M. Fast IR-Actuated Shape-Memory Polymers Using In Situ Silver Nanoparticle-Grafted Cellulose Nanocrystals. ACS Appl. Mater. Interfaces 2018, 10, 29933-29942.

(16) Yu, H.; Fang, D.; Dirican, M.; Wang, R.; Tian, Y.; Chen, L.; Liu, H.; Wang, J.; Tang, F.; Asiri, A. M.; Zhang, X.; Tao, J. Binding Conductive Ink Initiatively and Strongly: Transparent and Thermally Stable Cellulose Nanopaper as a Promising Substrate for Flexible Electronics. ACS Appl. Mater. Interfaces 2019, 11, 20281-20290.

(17) Li, J.; Kang, L.; Wang, B.; Chen, K.; Tian, X.; Ge, Z.; Zeng, J.; $\mathrm{Xu}, \mathrm{J}$; $\mathrm{Gao}, \mathrm{W}$. Controlled Release and Long-Term Antibacterial Activity of Dialdehyde Nanofibrillated Cellulose/Silver Nanoparticle Composites. ACS Sustainable Chem. Eng. 2018, 7, 1146-1158.

(18) Zhao, S.; Caruso, F.; Dähne, L.; Decher, G.; De Geest, B. G.; Fan, J.; Feliu, N.; Gogotsi, Y.; Hammond, P. T.; Hersam, M. C.; Khademhosseini, A.; Kotov, N.; Leporatti, S.; Li, Y.; Lisdat, F.; LizMarzán, L. M.; Moya, S.; Mulvaney, P.; Rogach, A. L.; Roy, S.; Shchukin, D. G.; Skirtach, A. G.; Stevens, M. M.; Sukhorukov, G. B.; Weiss, P. S.; Yue, Z.; Zhu, D.; Parak, W. J. The Future of Layer-byLayer Assembly: A Tribute to ACS Nano Associate Editor Helmuth Möhwald. ACS Nano 2019, 13, 6151-6169.

(19) Szweda, R.; Tschopp, M.; Felix, O.; Decher, G.; Lutz, J. F. Sequences of Sequences: Spatial Organization of Coded Matter through Layer-by-Layer Assembly of Digital Polymers. Angew. Chem., Int. Ed. 2018, 57, 15817-15821.

(20) Sarkar, K.; Braden, E. V.; Pogorzalek, S.; Yu, S.; Roth, S. V.; Müller-Buschbaum, P. Monitoring Structural Dynamics of in situ Spray-Deposited Zinc Oxide Films for Application in Dye-Sensitized Solar Cells. ChemSusChem 2014, 7, 2140-2145.

(21) Zhang, P.; Santoro, G.; Yu, S.; Vayalil, S. K.; Bommel, S.; Roth, S. V. Manipulating the Assembly of Spray-Deposited Nanocolloids: In Situ Study and Monolayer Film Preparation. Langmuir 2016, 32, 4251-4258.

(22) Roth, S. V.; Herzog, G.; Körstgens, V.; Buffet, A.; Schwartzkopf, M.; Perlich, J.; Abul Kashem, M. M.; Döhrmann, R.; Gehrke, R.; Rothkirch, A.; Stassig, K.; Wurth, W.; Benecke, G.; Li, C.; Fratzl, P.; Rawolle, M.; Müller-Buschbaum, P. In Situ Observation of Cluster Formation during Nanoparticle Solution Casting on a Colloidal Film. J. Phys.: Condens. Matter 2011, 23, 254208.

(23) Al-Hussein, M.; Herzig, E. M.; Schindler, M.; Löhrer, F.; Palumbiny, C. M.; Wang, W.; Roth, S. V.; Müller-Buschbaum, P. Comparative Study of the Nanomorphology of Spray and Spin Coated PTB7 Polymer: Fullerene films. Polym. Eng. Sci. 2016, 56, 889-894.

(24) Roth, S. V. A Deep Look into the Spray Coating Process in Real-Time-the Crucial Role of X-Rays. J. Phys.: Condens. Matter 2016, 28, 403003.

(25) Geng, L.; Mittal, N.; Zhan, C.; Ansari, F.; Sharma, P. R.; Peng, X.; Hsiao, B. S.; Söderberg, L. D. Understanding the Mechanistic Behavior of Highly Charged Cellulose Nanofibers in Aqueous Systems. Macromolecules 2018, 51, 1498-1506.

(26) Brett, C. J.; Mittal, N.; Ohm, W.; Gensch, M.; Kreuzer, L. P.; Körstgens, V.; Månsson, M.; Frielinghaus, H.; Müller-Buschbaum, P.; Söderberg, L. D.; Roth, S. V. Water-Induced Structural Rearrangements on the Nanoscale in Ultrathin Nanocellulose Films. Macromolecules 2019, 52, 4721-4728. 
(27) Ohm, W.; Rothkirch, A.; Pandit, P.; Körstgens, V.; MüllerBuschbaum, P.; Rojas, R.; Yu, S.; Brett, C. J.; Söderberg, D. L.; Roth, S. V. Morphological Properties of Airbrush Spray-Deposited Enzymatic Cellulose Thin Films. J. Coat. Technol. Res. 2018, 15, 759-769.

(28) Perito, B.; Giorgetti, E.; Marsili, P.; Muniz-Miranda, M. Antibacterial Activity of Silver Nanoparticles Obtained by Pulsed Laser Ablation in Pure Water and in Chloride Solution. Beilstein J. Nanotechnol. 2016, 7, 465-473.

(29) Reich, S.; Letzel, A.; Menzel, A.; Kretzschmar, N.; Gökce, B.; Barcikowski, S.; Plech, A. Early Appearance of Crystalline Nanoparticles in Pulsed Laser Ablation in Liquids Dynamics. Nanoscale 2019, 11, 6962-6969.

(30) Khapli, S.; Rianasari, I.; Blanton, T.; Weston, J.; Gilardetti, R.; Neiva, R.; Tovar, N.; Coelho, P. G.; Jagannathan, R. Fabrication of Hierarchically Porous Materials and Nanowires through Coffee Ring Effect. ACS Appl. Mater. Interfaces 2014, 6, 20643-20653.

(31) Buffet, A.; Rothkirch, A.; Döhrmann, R.; Körstgens, V.; Abul Kashem, M. M.; Perlich, J.; Herzog, G.; Schwartzkopf, M.; Gehrke, R.; Müller-Buschbaum, P.; Roth, S. V. P03, the Microfocus and Nanofocus X-Ray Scattering (Minaxs) Beamline of the Petra III Storage Ring: the Microfocus Endstation. J. Synchrotron Radiat. 2012, 19, 647-653.

(32) Benecke, G.; Wagermaier, W.; Li, C.; Schwartzkopf, M.; Flucke, G.; Hoerth, R.; Zizak, I.; Burghammer, M.; Metwalli, E.; MüllerBuschbaum, P.; Trebbin, M.; Förster, S.; Paris, O.; Roth, S. V.; Fratzl, P. A Customizable Software for Fast Reduction and Analysis of Large X-Ray Scattering Data Sets: Applications of the New DPDAK Package to Small-Angle X-Ray Scattering and Grazing-Incidence Small-Angle X-Ray Scattering. J. Appl. Crystallogr. 2014, 47, 1797-1803.

(33) Schaffer, C. J.; Palumbiny, C. M.; Niedermeier, M. A.; Jendrzejewski, C.; Santoro, G.; Roth, S. V.; Müller-Buschbaum, P. A Direct Evidence of Morphological Degradation on a Nanometer Scale in Polymer Solar Cells. Adv. Mater. 2013, 25, 6760-6764.

(34) Wang, W.; Song, L.; Magerl, D.; Moseguí González, D.; Körstgens, V.; Philipp, M.; Moulin, J.-F.; Müller-Buschbaum, P. Influence of Solvent Additive 1,8-Octanedithiol on P3HT:PCBM Solar Cells. Adv. Funct. Mater. 2018, 28, 1800209.

(35) Jiang, Z. GIXSGUI: a MATLAB Toolbox for Grazing-Incidence $X$-Ray Scattering Data Visualization and Reduction, and Indexing of Buried Three-Dimensional Periodic Nanostructured Films. J. Appl. Crystallogr. 2015, 48, 917-926.

(36) Horcas, I.; Fernández, R.; Gómez-Rodriguez, J. M.; Colchero, J.; Gómez-Herrero, J.; Baro, A. M. WSXM: A Software for Scanning Probe Microscopy and A Tool for Nanotechnology. Rev. Sci. Instrum. 2007, 78, No. 013705 .

(37) Soethoudt, J.; Grillo, F.; Marques, E. A.; van Ommen, J. R.; Tomczak, Y.; Nyns, L.; Van Elshocht, S.; Delabie, A. DiffusionMediated Growth and Size-Dependent Nanoparticle Reactivity during Ruthenium Atomic Layer Deposition on Dielectric Substrates. Adv. Mater. Interfaces 2018, 5, 1800870.

(38) Elzey, S.; Grassian, V. H. Nanoparticle Dissolution from the Particle Perspective: Insights from Particle Sizing Measurements. Langmuir 2010, 26, 12505-12508.

(39) Peretyazhko, T. S.; Zhang, Q.; Colvin, V. L. Size-Controlled Dissolution of Silver Nanoparticles at Neutral and Acidic $\mathrm{pH}$ Conditions: Kinetics and Size Changes. Environ. Sci. Technol. 2014, 48, 11954-11961.

(40) Pandit, P.; Schwartzkopf, M.; Rothkirch, A.; Roth, S. V.; Bernstorff, S.; Gupta, A. Structure-Function Correlations in Sputter Deposited Gold/Fluorocarbon Multilayers for Tuning Optical Response. Nanomaterials 2019, 9, 1249.

(41) Yoneda, Y. Anomalous Surface Reflection of X Rays. Phys. Rev. 1963, 131, 2010-2013.

(42) Yang, D.; Löhrer, F. C.; Körstgens, V.; Schreiber, A.; Cao, B.; Bernstorff, S.; Müller-Buschbaum, P. In Operando GISAXS and GIWAXS Stability Study of Organic Solar Cells Based on PffBT4T2OD:PC71BM with and without Solvent Additive. Adv. Sci. 2020, 7, 2001117.
(43) Chen, W.; Tang, H.; Li, N.; Scheel, M. A.; Xie, Y.; Li, D.; Körstgens, V.; Schwartzkopf, M.; Roth, S. V.; Wang, K.; Sun, X. W.; Müller-Buschbaum, P. Colloidal PbS Quantum Dot Stacking Kinetics during Deposition via Printing. Nanoscale Horiz. 2020, 5, 880-885.

(44) Wienhold, K. S.; Weindl, C. L.; Yin, S.; Tian, T.; Schwartzkopf, M.; Rothkirch, A.; Roth, S. V.; Müller-Buschbaum, P. Following In Situ the Evolution of Morphology and Optical Properties during Printing of Thin Films for Application in Non-Fullerene Acceptor Based Organic Solar Cells. ACS Appl. Mater. Interfaces 2020, 12, 40381-40392.

(45) Zhao, Y.; Kornienko, N.; Liu, Z.; Zhu, C.; Asahina, S.; Kuo, T. R.; Bao, W.; Xie, C.; Hexemer, A.; Terasaki, O.; Yang, P.; Yaghi, O. M. Mesoscopic Constructs of Ordered and Oriented Metal-Organic Frameworks on Plasmonic Silver Nanocrystals. J. Am. Chem. Soc. 2015, 137, 2199-2202.

(46) Raza, M. A.; Kanwal, Z.; Rauf, A.; Sabri, A. N.; Riaz, S.; Naseem, S. Size- and Shape-Dependent Antibacterial Studies of Silver Nanoparticles Synthesized by Wet Chemical Routes. Nanomaterials 2016, 6, 74

(47) Bushroa, A. R.; Rahbari, R. G.; Masjuki, H. H.; Muhamad, M. R. Approximation of Crystallite Size and Microstrain via XRD Line Broadening Analysis in TiSiN Thin Films. Vacuum 2012, 86, 11071112.

(48) Smilgies, D. M. Scherrer Grain-Size Analysis Adapted to Grazing-Incidence Scattering with Area Detectors. J. Appl. Crystallogr. 2009, 42, 1030-1034.

(49) Li, H.; Li, A.; Zhao, Z.; Li, M.; Song, Y. Heterogeneous Wettability Surfaces: Principle, Construction, and Applications. Small Struct. 2020, 1, 2000028.

(50) Li, Y.; Chen, L.; Wooding, J. P.; Zhang, F.; Lively, R. P.; Ramprasad, R.; Losego, M. D. Controlling Wettability, Wet Strength, and Fluid Transport Selectivity of Nanopaper with Atomic Layer Deposited (ALD) Sub-Nanometer Metal Oxide Coatings. Nanoscale Adv. 2020, 2, 356-367.

(51) Li, D.; Wu, J.; Yang, S.; Zhang, W.; Niu, X.; Chen, Y.; Ran, F. Hydrophilicity and Anti-Fouling Performance of Polyethersulfone Membrane Modified by Grafting Block Glycosyl Copolymers via Surface Initiated Electrochemically Mediated Atom Transfer Radical Polymerization. New J. Chem. 2018, 42, 2692-2701.

(52) Wang, Y.; Gong, X. Special Oleophobic and Hydrophilic Surfaces: Approaches, Mechanisms, and Applications. J. Mater. Chem. A 2017, 5, 3759-3773.

(53) Zhang, T.; Fang, L.; Lin, N.; Wang, J.; Wang, Y.; Wu, T.; Song, P. Highly Transparent, Healable, and Durable Anti-Fogging Coating by Combining Hydrophilic Pectin and Tannic Acid with Poly(Ethylene Terephthalate). Green Chem. 2019, 21, 5405-5413.

(54) Kats, M. A.; Capasso, F. Optical Absorbers Based on Strong Interference in Ultra-Thin Films. Laser Photonics Rev. 2016, 10, 735749.

(55) Pérez-González, O.; Zabala, N.; Aizpurua, J. Optical Characterization of Charge Transfer and Bonding Dimer Plasmons in Linked Interparticle Gaps. New J. Phys. 2011, 13, No. 083013.

(56) Kim, D.-S.; Heo, J.; Ahn, S.-H.; Han, S. W.; Yun, W. S.; Kim, Z. $\mathrm{H}$. Real-Space Mapping of The Strongly Coupled Plasmons of Nanoparticle Dimers. Nano Lett. 2009, 9, 3619-3625.

(57) Valmalette, J. C.; Tan, Z.; Abe, H.; Ohara, S. Raman Scattering of Linear Chains of Strongly Coupled Ag Nanoparticles on SWCNTS. Sci. Rep. 2014, 4, 5238.

(58) Schürmann, U.; Takele, H.; Zaporojtchenko, V.; Faupel, F. Optical and Electrical Properties of Polymer Metal Nanocomposites Prepared by Magnetron Co-Sputtering. Thin Solid Films 2006, 515, 801-804.

(59) Bellet, D.; Lagrange, M.; Sannicolo, T.; Aghazadehchors, S.; Nguyen, V. H.; Langley, D. P.; Muñoz-Rojas, D.; Jiménez, C.; Bréchet, Y.; Nguyen, N. D. Transparent Electrodes Based on Silver Nanowire Networks: From Physical Considerations towards Device Integration. Materials 2017, 10, 570.

(60) Rey-García, F.; Flores-Arias, M. T.; Gómez-Reino, C.; Lahoz, R.; de la Fuente, G. F.; Assenmacher, W.; Mader, W. Microstructure 
of Planar Glass Substrates Modified by Laser Ablation Backwriting (LAB) of Metal Targets. Appl. Surf. Sci. 2014, 307, 645-653.

(61) Etrich, C.; Fahr, S.; Hedayati, M.; Faupel, F.; Elbahri, M.; Rockstuhl, C. Effective Optical Properties of Plasmonic Nanocomposites. Materials 2014, 7, 727-741.

(62) Reinhard, B. M.; Siu, M.; Agarwal, H.; Alivisatos, A. P.; Liphardt, J. Calibration of Dynamic Molecular Rulers Based on Plasmon Coupling between Gold Nanoparticles. Nano Lett. 2005, 5, $2246-2252$.

(63) Sarina, S.; Waclawik, E. R.; Zhu, H. Photocatalysis on Supported Gold and Silver Nanoparticles under Ultraviolet and Visible Light Irradiation. Green Chem. 2013, 15, 1814-1833.

(64) Anker, J. N.; Hall, W. P.; Lyandres, O.; Shah, N. C.; Zhao, J.; Van Duyne, R. P. Biosensing with Plasmonic Nanosensors. Nat. Mater. 2008, 7, 442-453.

(65) Yu, Z.; Wang, W.; Dhital, R.; Kong, F.; Lin, M.; Mustapha, A. Antimicrobial Effect and Toxicity of Cellulose Nanofibril/Silver Nanoparticle Nanocomposites Prepared by an Ultraviolet Irradiation Method. Colloids Surf., B 2019, 180, 212-220.

(66) Eskilson, O.; Lindström, S. B.; Sepulveda, B.; Shahjamali, M. M.; Güell-Grau, P.; Sivlér, P.; Skog, M.; Aronsson, C.; Björk, E. M.; Nyberg, N.; Khalaf, H.; Bengtsson, T.; James, J.; Ericson, M. B.; Martinsson, E.; Selegård, R.; Aili, D. Self-Assembly of Mechanoplasmonic Bacterial Cellulose-Metal Nanoparticle Composites. Adv. Funct. Mater. 2020, 30, 2004766.

(67) Xu, K.; Zhou, R.; Takei, K.; Hong, M. Toward Flexible SurfaceEnhanced Raman Scattering (SERS) Sensors for Point-of-Care Diagnostics. Adv. Sci. 2019, 6, 1900925. 